\title{
Evaluation of tumor-free distance and depth of myometrial invasion as prognostic factors in endometrial cancer
}

\author{
RAOUDHA DOGHRI, SALMA CHAABOUNI, YOLDEZ HOUCINE, LAMIA CHARFI, \\ NADIA BOUJELBENE, MAHA DRISS and KARIMA MRAD \\ Pathology Department, Salah Azaïz Institution, 1006 Tunis, Tunisia
}

Received November 14, 2017; Accepted April 12, 2018

DOI: $10.3892 / \mathrm{mco} .2018 .1629$

\begin{abstract}
The aim of the study was to investigate whether the tumor free distance (TFD), which is the distance in millimeters between the deepest point of invasion and the serosal surface, and absolute depth of invasion (DMI), the distance in millimeters between the endomyometrial junction and the deepest point of myometrial invasion, are useful in surgical staging and in predicting prognosis. The present study retrospectively analyzed 62 cases of endometrial carcinoma with complete surgical staging, carried out over a 4 and half-year period (January 2003 to June 2007). All surgicopathological findings including surgical stages, histological type and grade, myometrial invasion, lymphovascular space invasion, cervical and adnexal involvement, and lymph node metastasis were abstracted from medical records and pathological reports. Univariate and multivariate analyses were performed comparing TFD, DMI and the percentage of mypmetrial invasion (MI) with established prognostic factors. A total of 62 patients were included in the study. A total of $52(84 \%)$ had endometrioid carcinomas and 31 patients $(60 \%)$ had grade 1 cancer. The deepest MI was $<50 \%$ in 32 patients (52\%). Median DMI was $2.7 \mathrm{~mm}$ (range 0-15 mm). Median TFD was $3 \mathrm{~mm}$ (range 0-19 $\mathrm{mm}$ ). There was lymphovascular space invasion (LVSI) in 11 patients $(17.5 \%)$, cervical involvement in 11 patients (17.5\%), extra-uterine extension in 9 cases (14\%) and lymph node metastasis in 12 patients (22\%). It was demonstrated that $50 \%$ MI was significantly associated with prognostic factors (cervical involvement, type 2 carcinomas and LVSI, and was a significant predictor of the 5-year overall survival rate and recurrence-free survival $(\mathrm{P}=0.05, \mathrm{P}=0.01)$. No significant association was observed between DMI and TFD with clinicopathological parameters and survival rates. The importance of DMI in predicting recurrence of disease was observed to be highest in terms of sensitivity and
\end{abstract}

Correspondence to: Dr Yoldez Houcine, Pathology Department, Salah Azaïz Institution, Bab Saadoun Street, 1006 Tunis, Tunisia E-mail: daddoussa87@hotmail.fr

Key words: endometrium cancer, myometrial invasion, tumor-free distance, cancer staging, prognosis specificity. The cut-off value with the highest sensitivity and specificity crossing the receive operating characteristic curve was calculated to be $3 \mathrm{~mm}$ for DMI and $2.5 \mathrm{~mm}$ for TFD. The results indicate that DMI is a superior predictive factor of recurrence of the disease compared with TFD. However, further studies are required in order to prove the prognostic usefulness of these parameters and then to improve management of endometrial cancer.

\section{Introduction}

Endometrial carcinoma is the most common of all the gynecologic malignancies and its incidence is rising $(1,2)$. Surgical staging is an important determinant of adjuvant therapy. Whilst most patients with early stage disease have a good prognosis, the prognosis for patients with recurrent or advanced disease at presentation, i.e., advanced stage, incurable by surgery or radiotherapy, is poor. The most important parameter to elaborate the surgical stage is the depth of myometrial invasion $(3,4)$. The percentage of myometrial invasion $(\% \mathrm{MI})$ is routinely determined in hysterectomy specimens and categorized as greater than or less than $50 \%$ and it is one of the parameters used in the determination of the need for adjuvant therapy (5). However, more recently, two other alternative methods of measuring myometrial invasion have been proposed: Absolute depth of invasion (DMI, the distance in millimeters between the endomyometrial junction and the deepest point of myometrial invasion) and tumour-free distance from serosa (TFD, the distance in millimeters between the deepest point of invasion and the serosal surface) (6). Previous studies comparing the predictive value of TFD and DMI were underpowered (7).

Our objectives were: i) to compare the predictive prognostic value of TFD, DMI and 50\% MI; and ii) to evaluate the relationship of these parameters with clinicopathologic factors and progression free survival.

\section{Patients and methods}

The present study is retrospective carried out over a 4 and half-year period (January 2003 and June 2007) and conducted at the Pathology Department of Salah Azaeiz Institute, Tunisia. Patients treated surgically by hysterectomy and bilateral salpingo-oophorectomy with or without pelvic lymphadenectomy for primary endometrial carcinoma were 
recorded. A total of 62 cases were identified. Clinical information including: Age at diagnosis, follow-up and survival data (date of recurrence, date of death and the cause of death) were collected from medical records. Surgical data were abstracted from operatory reports. Stage of disease was defined according to the 2009 International Federation of Gynecology and Obstetrics (FIGO) staging system (8). Adjuvant curietherapy was indicated for all stages. External radiotherapy was indicated for stage IA type 2 or with vascular invasion, stage IB grade 3 and stages II, III and IV.

The slides of the primary endometrial carcinoma of all patients were reviewed by a specialist gynaecological pathologist to determine histological type, tumor grade, myometrial thickness, DMI, TFD, \%MI categorized as more or less than $50 \%(50 \% \mathrm{MI})$, lymphovascular space invasion (LVSI), cervical involvement, serosal and adnexal involvement and lymph node status. Myometrial thickness was measured from the endomyometrial junction to the serosal surface. TFD was measured from the deepest point of invasion to the serosal surface. DMI was defined as the distance between the endomyometrial junction and the deepest point of myometrial invasion. If there confounding factors were present such as the irregularity of the endomyometrial junction, the extension of the tumor into adenomyotic foci, exophytic tumors and extensive smooth muscle metaplasia within the stroma of the neoplasm, slides were reviewed by another specialist gynecological pathologist, blind to any outcome data. Between 3 and 6 slides of tumor were reviewed for each case. The \%MI was calculated from the equation: $\mathrm{MI}=(\mathrm{DMIx} 100) /(\mathrm{DMI}+\mathrm{RFD})$. Follow up was to the last date seen in the outpatients' clinics.

Statistical analysis was performed using the software package SPSS 16.0 for Microsoft Windows. Receiver operating characteristics (ROC) analyses were established to evaluate the performance (sensitivity and specificity) of DMI and TFD to predict recurrence disease. Univariate and multivariate cox regression analyses were performed to correlate between DMI, TFD, 50\% MI and clinicopathologic characteristics (age, histologic type, tumor grade, FIGO stage, LVSI, cervical involvement, extra-uterine involvement and lymph node status). Survival analysis was calculated using the method of Kaplan Meier and was then correlated with DMI, TFD, 50\% MI and other clinicopathologic characteristics by Log Rank test.

\section{Results}

Patient characteristics are summarized in Table I. The mean age of 62 patients enrolled in the study was 60 years and the majority of patients (86\%) were in the postmenopausal period. According to the last WHO Classification of endometrial cancer (9), histology revealed endometrioid carcinoma in 52 cases $(84 \%)$, serous carcinoma (6 cases) and clear cell carcinoma (4 cases). Thirty one patients $(60 \%)$ had grade 1 cancer, 18 patients $(34 \%)$ had grade 2,13 patients $(20 \%)$ had grade 3. Myometrial invasion was found in 87 cases (92\%). The deepest myometrial invasion was less than $50 \%$ in 32 patients $(52 \%)$. Median DMI was 2,7 mm (range 0-15 mm). Median TFD was $3 \mathrm{~mm}$ (range $0-19 \mathrm{~mm}$ ). There was LVSI in 11 patients $(17,5 \%)$, cervical involvement in 11 patients $(17,5 \%)$, extra-uterine extension in 9 cases (14\%) and lymph node metastasis in 12 patients $(22 \%)$. Patient characteristics are shown in Table I.

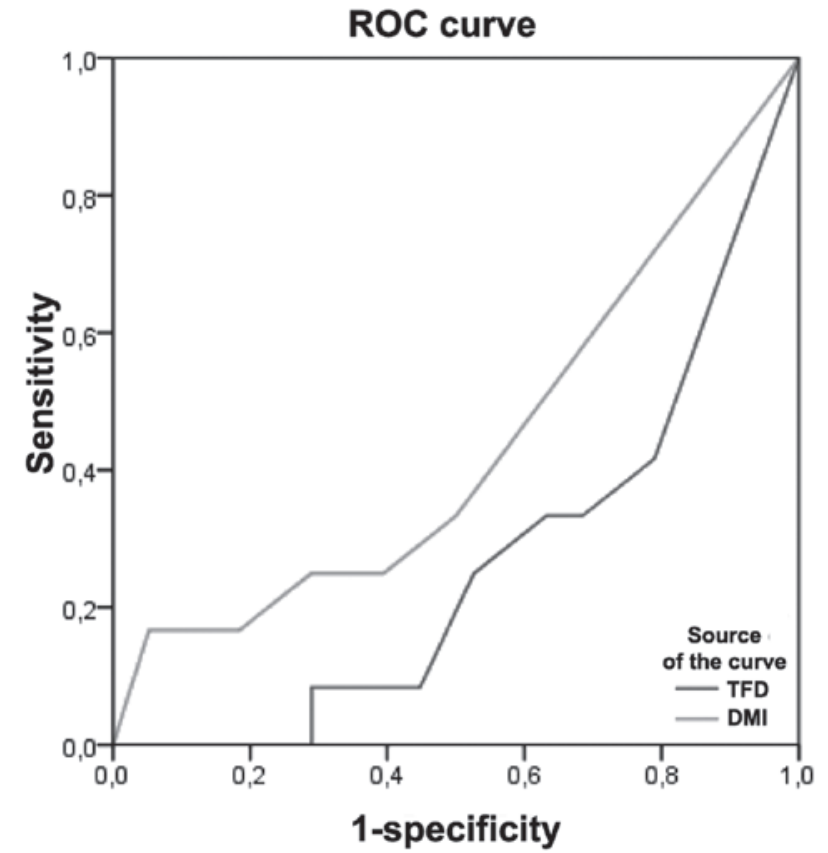

Figure 1. Highest sensitivity and specificity of depth of myometrial invasion in predicting recurrence of disease.

The majority was diagnosed with FIGO stage I (35 patients). Total hysterectomy with bilateral annexectomy was performed in all patients. Lymphadenectomy was indicated in 54 cases. Forty four patients received adjuvant therapy. The median follow-up time was 3, 8 years (1-9 years), with 10 deaths $(16 \%)$ and 12 recurrences $(19,3 \%)$.

ROC curve. DMI showed a larger area under the ROC curve (AUC) than TFD for prediction of recurrence of disease: 0 , 43 and 0,26 respectively. Thus, The importance of DMI in predicting recurrence of disease was found to be highest in terms of sensitivity and specificity (Fig. 1). The cut-off value with the highest sensitivity and specificity crossing the ROC curve was calculated to be $3 \mathrm{~mm}$ for DMI and 2,5 $\mathrm{mm}$ for TFD.

Correlation between 50\% MI, DMI and TFD with clinicopathologic parameters. In univariable analysis, $>50 \% \mathrm{MI}$ was significantly associated with cervical involvement, type II carcinomas and LVSI (Table II). No significant association was found between DMI and TFD with clinicopathologic parameters.

Survival analyses. In univariate analysis, only percentage of myometrial invasion $(50 \% \mathrm{MI})$ was significant predictor of the 5-year overall survival rate and recurrence-free survival $(\mathrm{P}=0.05, \mathrm{P}=0.01)$ (Tables III and IV). No significant association was found between DMI and TFD with survival rates.

\section{Discussion}

Our study show different result from earlier studies in proposing TFD and DMI as a useful measure of myometrial invasiveness.

The prognostic significance of MI is well documented (10). It is associated with recurrence, overall survival, and lymph 
Table I. Clinical and pathologic characteristics of all patients $(n=62)$.

Variable
Age
Stade (FIGO 2009
IA
IB
II
III
IV
Histologic type
Endometrioid
Serous
Clear cell

Histogenetic type

Type 1

Type 2

Range (\%)

Histologic grade

G1

G2

G3

$\mathrm{N} / \mathrm{m}$

52

Tumour size, $\mathrm{mm}$

$<20$

$\geq 20$

Non precised

Myometrial invasion

Absent, \%

$\geq 50$

$<50$

DMI, mm

$\geq 3$

$<3$

TFD, $\mathrm{mm}$

$\geq 3$

$<3$

40

22

Cervical involvement

Yes

No

45

LVSI

Yes

No

Lymph node involvement

Yes

No

Extrauterine involvement

Yes

Adjuvant therapy

No

Curietherapy

Radiotherapy

Radio+Curietherapy
26

9

10

11

6

6

4

52

10

31

18

13

34

19

5

25

32

17

40-87

42

14

16

18

10

84

10

6

84

16

50

29

21

9

14

56

30

8

40

52

47

53

64,5

35,5

27

73

17.5

82.5

51

2

22

73

14

86

29

37

13

13
Table I. Continued.

\begin{tabular}{lcc}
\hline Variable & N/median & Range $(\%)$ \\
\hline Recurrence & 4 & \\
Yes & 58 & 6.5 \\
No & & 93.5 \\
Death & 12 & \\
Yes & 50 & 19.5 \\
No & & 80.5 \\
\hline
\end{tabular}

Table II. The association between presence of $\geq 1 / 2$ myometrial invasion (MI), the depth of myometrial invasion (DMI), tumor-free distance from Serosa and prognostic.

\begin{tabular}{lll}
\hline Variable & $>\%$ MI & DMI \\
\hline Stage & $\mathbf{P}=\mathbf{0 . 0 0 0}$ & $\mathbf{P}=\mathbf{0 . 0 0 2}$ \\
Histologic grade & $\mathrm{P}=0.077$ & $\mathrm{P}=0.56$ \\
Histologic type & $\mathbf{P}=\mathbf{0 . 0 3 2}$ & $\mathrm{P}=0.13$ \\
Lymphovascular & $\mathbf{P}=\mathbf{0 . 0 0 3}$ & $\mathrm{P}=0.82$ \\
space invasion & & $\mathrm{P}=0.38$ \\
Cervical involvement & $\mathbf{P}=\mathbf{0 . 0 0 4}$ & $\mathrm{P}=0.29$ \\
Lymphadenectomy & $\mathrm{P}=0.29$ & $\mathrm{P}=0.42$ \\
Lymph node involvement & $\mathrm{P}=0.19$ & \\
Extrauterine extension & $\mathrm{P}=0.24$ & $\mathrm{P}=0.39$ \\
\hline
\end{tabular}

Bold print denotes statistical significance.

Table III. Correlation between 5-year overall survival and clinicopathologic parameters.

\begin{tabular}{lcc}
\hline Variable & $\begin{array}{c}\text { 5-year overall } \\
\text { survival (\%) }\end{array}$ & P-value \\
\hline $\begin{array}{l}\text { Myometrial invasion } \\
\geq 50 \%\end{array}$ & 87 & \\
$<50 \%$ & 72 & 0.05 \\
DOI, mm & & \\
$\geq 3$ & 83 & 0.57 \\
$<3$ & 79 & \\
TFD, mm & & 0.17 \\
$\geq 3$ & 88 & \\
$<3$ & 74 & \\
\hline
\end{tabular}

node involvement (11). In this study, the ability of 50\% MI, DMI and TFD to predict recurrence and death from disease was investigated. DMI showed a larger area under the ROC curve for prediction of recurrence than TFD. Our results revealed that a cut-off value of $3 \mathrm{~mm}$ DMI and 2,5 $\mathrm{mm}$ TFD led to the most effective balance between sensitivity and specificity. For DMI, only one study proposed a cut-off value of $4 \mathrm{~mm}$ and showed that DMI was a better predictor of 
Table IV. Correlation between 5-year recurrence-free survival and clinicopathologic parameters.

\begin{tabular}{|c|c|c|}
\hline Variable & $\begin{array}{l}\text { 5-year recurrence- } \\
\text { free survival }(\%)\end{array}$ & P-value \\
\hline \multicolumn{3}{|l|}{ Age, years } \\
\hline$\leq 50$ & 72 & \multirow[t]{2}{*}{0.81} \\
\hline$>50$ & 69 & \\
\hline \multicolumn{3}{|l|}{ Stage } \\
\hline IA & 81 & \multirow[t]{5}{*}{0.000} \\
\hline IB & 100 & \\
\hline II & 80 & \\
\hline III & 63 & \\
\hline IV & 0 & \\
\hline \multicolumn{3}{|c|}{ Lymphadenectomy } \\
\hline Yes & 46 & \multirow[t]{2}{*}{0.017} \\
\hline No & 77 & \\
\hline \multicolumn{3}{|l|}{ Histologic type } \\
\hline Endometrioid & 74 & \multirow[t]{3}{*}{0.66} \\
\hline Serous & 60 & \\
\hline Clear cell & 75 & \\
\hline \multicolumn{3}{|l|}{$\begin{array}{l}\text { Lymphovascular } \\
\text { space invasion }\end{array}$} \\
\hline Yes & 54 & \multirow[t]{2}{*}{0.09} \\
\hline No & 77 & \\
\hline \multicolumn{3}{|l|}{ Histologic grade } \\
\hline G1 & 74 & \multirow[t]{3}{*}{0.078} \\
\hline $\mathrm{G} 2$ & 84 & \\
\hline G3 & 53 & \\
\hline \multicolumn{3}{|c|}{ Myometrial invasion, \% } \\
\hline$\geq 50$ & 55 & \multirow[t]{2}{*}{0.01} \\
\hline$<50$ & 82 & \\
\hline \multicolumn{3}{|l|}{ DOI, mm } \\
\hline$\geq 3$ & 65 & \multirow[t]{2}{*}{0.17} \\
\hline$<3$ & 85 & \\
\hline \multicolumn{3}{|c|}{ Median TFD, mm } \\
\hline$\geq 3$ & 64 & \multirow[t]{2}{*}{0.17} \\
\hline$<3$ & 82 & \\
\hline \multicolumn{3}{|c|}{ Cervical involvement } \\
\hline Yes & 70 & \multirow[t]{2}{*}{0.79} \\
\hline No & 75 & \\
\hline \multicolumn{3}{|c|}{ Lymph node involvement } \\
\hline Yes & 56 & \multirow[t]{2}{*}{0.002} \\
\hline No & 82 & \\
\hline \multicolumn{3}{|l|}{ Radiotherapy } \\
\hline Yes & 59 & \multirow[t]{2}{*}{$\mathrm{P}=0.14$} \\
\hline No & 78 & \\
\hline \multicolumn{3}{|l|}{ Curietherapy } \\
\hline Yes & 82 & 0.003 \\
\hline No & 51 & \\
\hline
\end{tabular}

recurrence than TFD, and was more strongly correlated with clinicopathologic parameters than TFD and 50\% MI (12).
The concept of DMI as a better prognostic indicator than TFD and 50\% MI has been documented in the study of Kondalsamy-Chennakesavan et al (6). In fact, authors showed that depth of MI can predict nodal involvement independently when compared with TFD. On the other hand, several studies have shown that TFD is superior than DMI and 50\% MI in predicting disease extension as well as outcome $(11,13)$. Chattopadhyay et al (10) demonstrated that TFD is an independent predictor of disease specific survival, recurrence and pelvic lymph node involvement. Data regarding the cut-off value of TFD in patients with endometrial cancer which revealed the best predictive performance value varied between 1,75 and $10 \mathrm{~mm}(7,14)$. In the current study, only $>50 \%$ MI was found to have a predictive value for cervical involvement, type 2 and LVSI. There was no statistically significant result for the correlation between DOI, TFD and clinicopathologic parameters. As far as the reproductibility of measurement of myometrial invasion is concerned, the best agreement is reached when measuring \%MI and TFD according to van der Putten et al (14). Myometrial invasion is sometimes affected by confounding factors, including the irregularity of the endomyometrial junction, the extension of the tumour into adenomyotic foci, exophytic tumours, leiomyomas and different patterns of myometrial invasion $(15,16)$. On the other hand, TFD, defined as the distance from deepest myometrial invasion to the serosal surface, is a way of assessing the myometrial invasiveness of the tumour that is not dependent on myometrial thickness and consequently is associated with low inter-observer variability (13). In our study, a discrepancy between \%MI and DMI was found in 11 cases $(17,7 \%)$. The most common reason for underestimation of myometrial invasion ( 7 cases) was intramural leiomyomas, and for overestimation (4 cases) was an exophytic tumour growth.

To our knowledge, this is the first study demonstrating the superiority of MI compared to TFD and DMI in predicting EC outcomes. This can be explained by the reduced number of patients included in this study compared with other study $(11,14)$. Besides, the lack of equitable distribution of histological types with a large predominance of endometroid carcinoma seems to influence the results of the study. Another confounding factor that must be mentioned is the short duration of follow up which influenced survival rates.

The shortcoming of this study is retrospectivity, while the evaluation requires larger prospective studies to clarify the impact of DMI and TFD.

In conclusion, our results indicate that DMI is a superior predictive factor of recurrence of disease compared to TFD. We need further studies to prove the prognostic usefulness of these parameters and then to improve management of endometrial cancer.

\section{Acknowledgements}

The authors would like to thank the Ministry of Health of Tunisia for its support.

\section{Funding}

No funding was received. 


\section{Availability of data and materials}

Not applicable.

\section{Authors' contributions}

$\mathrm{RD}$, revision of the manuscript and statistical analysis; SC, redaction the manuscript; $\mathrm{YH}$, redaction of the manuscript; LC, pathological diagnosis; NB, revision of the manuscript; MD, pathological diagnosis; KM, general supervision.

\section{Ethics approval and consent to participate}

Not applicable.

\section{Consent for publication}

Not applicable.

\section{Competing interests}

The authors declare that they have no competing interests.

\section{References}

1. Siegel RL, Miller KD and Jemal A: Cancer statistics, 2016. CA Cancer J Clin 66: 7-30, 2016.

2. Amant F, Mirza MR, Koskas M and Creutzberg CL: Cancer of the corpus uteri. Int J Gynaecol Obstet 131 (Suppl 2): S96-S104, 2015.

3. Vargas R, Rauh-Hain JA, Clemmer J, Clark RM, Goodman A, Growdon WB, Schorge JO, Del Carmen MG, Horowitz NS and Boruta DM II: Tumor size, depth of invasion and histologic grade as prognostic factors of lymph node involvement in endometrial cancer: A SEER analysis. Gynecol Oncol 133: 216-220, 2014.

4. Rahatli S, Dizdar O, Kucukoztas N, Oguz A, Yalcin S, Ozen O, Reyhan NH, Tarhan C, Yildiz F, Dursun P, et al: Good outcomes of patients with stage IB endometrial cancer with surgery alone. Asian Pac J Cancer Prev 15: 3891-3893, 2014.

5. ASTEC/EN.5 Study Group, Blake P, Swart AM, Orton J, Kitchener H, Whelan T, Lukka H, Eisenhauer E, Bacon M, Tu D, et al: Adjuvant external beam radiotherapy in the treatment of endometrial cancer (MRC ASTEC and NCIC CTG EN.5 randomised trials): Pooled trial results, systematic review and meta-analysis. Lancet 373: 137-146, 2009.
6. Kondalsamy-Chennakesavan S, van Vugt S, Sanday K, Nicklin J, Land R, Perrin L, Crandon A and Obermair A: Evaluation of tumor-free distance and depth of myometrial invasion as prognostic factors for lymph node metastases in endometrial cancer. Int J Gynecol Cancer 20: 1217-1221, 2010.

7. AJCC: AJCC Cancer Staging Manual. 6th edition. Springer Verlag, New York, 2002.

8. Zaino R, Carinelli SG, Ellenson LH, Eng C, Katabuchi H, Konishi I, Lax S, et al: Tumours of the uterine corpus: Epithelial tumours and precursors. In: WHO classification of tumours of female reproductive organs. 6th edition. Carcangiu ML, Herrington CS and Young RH (eds). World Health Organization, Kurman, RJ, pp126-132, 2014.

9. Schwab KV, O'Malley DM, Fowler JM, Copeland LJ and Cohn DE: Prospective evaluation of prognostic significance of the tumor-free distance from uterine serosa in surgically staged endometrial adenocarcinoma. Gynecol Oncol 112: 146-149, 2009.

10. Chattopadhyay S, Galaal KA, Patel A, Fisher A, Nayar A, Cross P and Naik R: Tumour-free distance from serosa is a better prognostic indicator than depth of invasion and percentage myometrial invasion in endometrioid endometrial cancer. BJOG 119: 1162-1170, 2012.

11. Geels YP, Pijnenborg JM, van den Berg-van Erp SH, Snijders MP Bulten J and Massuger LF: Absolute depth of myometrial invasion in endometrial cancer is superior to the currently used cut-off value of 50\%. Gynecol Oncol 129: 285-291, 2013.

12. Lindauer J, Fowler JM, Manolitsas TP, Copeland LJ, Eaton LA, Ramirez NC and Cohn DE: Is there a prognostic difference between depth of myometrial invasion and the tumor-free distance from the uterine serosa in endometrial cancer? Gynecol Oncol 91: 547-551, 2003.

13. Lee KB, Ki KD, Lee JM, Lee JK, Kim JW, Cho CH, Kim SM, Park SY, Jeong DH and Kim KT: The risk of lymph node metastasis based on myometrial invasion and tumor grade in endometrioid uterine cancers: A multicenter, retrospective Korean study. Ann Surg Oncol 16: 2882-2887, 2009.

14. van der Putten LJ, van de Vijver K, Bartosch C, Davidson B, Gatius S, Matias-Guiu X, McCluggage WG, Toledo G, van der Wurff AA, Pijnenborg JM, et al: Reproducibility of measurement of myometrial invasion in endometrial carcinoma. Virchows Arch 470: 63-68, 2017.

15. Quick CM, May T, Horowitz NS and Nucci MR: Low-grade, low-stage endometrioid endometrial adenocarcinoma: A clinicopathologic analysis of 324 cases focusing on frequency and pattern of myoinvasion. Int J Gynecol Pathol 31: 337-343, 2012.

16. Ali A, Black D and Soslow RA: Difficulties in assessing the depth of myometrial invasion in endometrial carcinoma. Int $\mathrm{J}$ Gynecol Pathol 26: 115-123, 2007. 